\title{
WEIGHTED NORM INEQUALITIES FOR HOMOGENEOUS SINGULAR INTEGRALS
}

\author{
JAVIER DUOANDIKOETXEA
}

\begin{abstract}
We prove weighted norm inequalities for homogeneous singular integrals when only a size condition is assumed on the restriction of the kernel to the unit sphere. The same results hold for the operator obtained by modifying the centered Hardy-Littlewood maximal operator over balls with a degree zero homogeneous function and also for the maximal singular integral.
\end{abstract}

\section{INTRODUCTION}

Given a function $\Omega$ over the unit sphere $S^{n-1}$ of $\mathbf{R}^{n}$, we will consider a singular integral operator $T_{\Omega}$ given by

$$
T_{\Omega} f(x)=\lim _{\varepsilon \rightarrow 0} \int_{|y|>\varepsilon} \frac{\Omega\left(y^{\prime}\right)}{|y|^{n}} f(x-y) d y
$$

and a related maximal operator

$$
M_{\Omega} f(x)=\sup _{r>0} \frac{1}{r^{n}} \int_{|y|<r}\left|\Omega\left(y^{\prime}\right) f(x-y)\right| d y,
$$

where $y^{\prime}=y|y|^{-1}$. The Calderón-Zygmund method of rotations [CZ] proves that if $\Omega \in L^{1}\left(S^{n-1}\right)$, then $M_{\Omega}$ is bounded in $L^{p}\left(\mathbf{R}^{n}\right), 1<p<\infty$, and the same is true for $T_{\Omega}$ if $\Omega$ is odd. If $\Omega$ is even, then $T_{\Omega}$ is bounded in $L^{p}\left(\mathbf{R}^{n}\right)$, $1<p<\infty$, when $\Omega \in L \log ^{+} L\left(S^{n-1}\right)$ (i.e., $|\Omega| \max (0, \log |\Omega|) \in L^{1}\left(S^{n-1}\right)$ ) and $\int_{S^{n-1}} \Omega=0$.

In this paper we are interested in weighted norm inequalities for $T_{\Omega}$ and $M_{\Omega}$; that is, we look for locally integrable nonnegative functions $w$ in $\mathbf{R}^{n}$ such that for some $C$ independent of $f$

$$
\int\left|T_{\Omega} f\right|^{p} w \leq C \int|f|^{p} w,
$$

and the same for $M_{\Omega}$. We denote by $L^{p}(w)$ the $L^{p}$ space with respect to the measure $w(x) d x$ in $\mathbf{R}^{n}$ so that (1) is equivalent to saying that $T_{\Omega}$ is a bounded operator in $L^{p}(w)$. The class of weights $w$ for which (1) holds for $T_{\Omega}\left(\right.$ resp. $\left.M_{\Omega}\right)$ will be denoted by $W_{p}\left(T_{\Omega}\right)$ (resp. $W_{p}\left(M_{\Omega}\right)$ ). Also, given $q$, the

Received by the editors November 13,1989 and, in revised form, April 17, 1990 and January 23, 1991.

1980 Mathematics Subject Classification (1985 Revision). Primary 42B20, 42B25.

The author was supported in part by DGICYT no. PB 86-108. 
class of weights $w$ for which (1) holds simultaneously for all $T_{\Omega}$ (resp. $M_{\Omega}$ ), $\Omega \in L^{q}\left(S^{n-1}\right)$ is denoted by $W_{p}\left(T_{q}\right)$ (resp. $W_{p}\left(M_{q}\right)$ ).

Weighted inequalities like (1) for $w(x)=|x|^{\alpha}$ are in [MW]. More general weights were studied in [KW1] but only assuming a Dini-type condition on $\Omega$. In this paper we show that the operator $T_{\Omega}$ is bounded in $L^{p}(w)$ for the weights $w$ considered in [KW1] and without assuming a Dini-type condition on $\Omega$. Also, we show that the same is true for $M_{\Omega}$. The technique we use is based on the alternative approach to the unweighted case presented in [DR1], where weighted inequalities for bounded $\Omega$ were also obtained. These results are proved in $\S 3$, after some technical lemmas given in $\S 2$. In $\S 4$ we study radial weights which include, for both $T_{\Omega}$ and $M_{\Omega}$, the weights of power type of [MW] for $T_{\Omega}$. In $\S 5$ some weights depending on $\Omega$ are also determined. In $\S 6$ we consider the maximal singular integral associated to $T_{\Omega}$ in order to deduce the almost everywhere convergence of the truncated integrals defining $T_{\Omega}$ for functions in $L^{p}(w)$. In $\S 7$ we extend the results to other singular integrals.

We recall that $A_{p}(p>1)$ is the class of weights $w$ for which the HardyLittlewood maximal function (corresponding to $\Omega \equiv 1$ and denoted throughout this paper by $M$ ) is bounded in $L^{p}(w)$ and was characterized by Muckenhoupt as those locally integrable nonnegative functions such that for some finite constant $C$ and any cube $Q$ in $\mathbf{R}^{n}$

$$
\left(\frac{1}{|Q|} \int_{Q} w\right)\left(\frac{1}{|Q|} \int_{Q} w^{-1 /(p-1)}\right)^{p-1} \leq C .
$$

$A_{1}$ is the class of weights $w$ for which $M$ satisfies a weak-type estimate in $L^{1}(w)$ and is characterized by $M w(x) \leq C w(x)$ a.e. For most of the results related to weighted norm inequalities we will refer to the monograph [GR] rather than to the original papers.

Notice finally that the inequality

$$
M_{\Omega} f(x) \leq C\left(M f^{q^{\prime}}(x)\right)^{1 / q^{\prime}} \quad\left(1 / q+1 / q^{\prime}=1\right)
$$

holds as a consequence of Hölder's inequality.

After this manuscript was ready the author learned that some of the results of this paper had been obtained independently by D. Watson (see [W]).

The author would like to thank the referee for his suggestions on improving the writing of the paper.

\section{SOME TECHNICAL LEMMAS}

Given $\Omega \in L^{q}\left(S^{n-1}\right), q>1$, we define

$$
E_{j} f(x)=2^{-j n} \int_{2^{j}<|y| \leq 2^{j+1}}\left|\Omega\left(y^{\prime}\right)\right| f(x-y) d y
$$

and if, moreover, $\int_{S^{n-1}} \Omega=0$, we also define

$$
T_{j} f(x)=\int_{2^{j}<|y| \leq 2^{j+1}} \frac{\Omega\left(y^{\prime}\right)}{|y|^{n}} f(x-y) d y .
$$

Then $M_{\Omega} f(x) \sim \sup _{j} E_{j}(|f|)(x)$ (i.e., their quotient is bounded above and below by positive finite constants);

$$
\left|T_{j} f(x)\right| \leq C E_{j}(|f|)(x) \text { and } T_{\Omega} f(x)=\sum_{j=-\infty}^{\infty} T_{j} f(x)
$$


for $f \in \mathscr{S}\left(\mathbf{R}^{n}\right)$. We also define

$$
g_{\Omega}(f)(x)=\left(\sum_{j=-\infty}^{\infty}\left|T_{j} f(x)\right|^{2}\right)^{1 / 2}
$$

and denote by $W_{p}\left(g_{\Omega}\right)$ the $L^{p}$-weights for $g_{\Omega}$.

Following [DR1] we make a new decomposition of $T_{\Omega}$. Choose a real function $\psi \in \mathscr{C}^{\infty}\left(\mathbf{R}^{n}\right)$, supported in $\frac{1}{2}<|\xi|<2$, and such that

$$
\sum_{k=-\infty}^{\infty}\left|\psi\left(2^{k} \xi\right)\right|^{2}=1 \quad \forall \xi \neq 0 .
$$

Define $S_{k}$ as $\left(S_{k} f\right)^{-}(\xi)=\psi\left(2^{k} \xi\right) \hat{f}(\xi)$ so that $\sum_{k} S_{k}^{2}=$ Id. Let $\tilde{T}_{k} f=$ $\sum_{j=-\infty}^{\infty} T_{j} S_{j+k}^{2} f ;$ then $T_{\Omega}=\sum_{k} \widetilde{T}_{k}$.

In [DR1] we proved that given $1<p<\infty$, there exists an $\alpha=\alpha(p)>0$ such that

$$
\left\|\widetilde{T}_{k} f\right\|_{p} \leq C 2^{-\alpha|k|}\|f\|_{p} .
$$

For $p=2$ this inequality is given by a Fourier transform estimate. In [DR1] it was shown that for any $p_{0} \neq 2$, (3) holds with a constant independent of $k$. Given $p$, take $p_{0}$ such that $p$ is between $p_{0}$ and 2 and interpolate to get (3). Alternatively, one could prove that the $H^{1}-L^{1}$ constant of $\widetilde{T}_{k}$ grows as $C(1+|k|)$ and apply interpolation again.

Lemma 1. Let $w \in A_{p}$. If the vector-valued inequality

$$
\left\|\left(\sum_{j}\left|E_{j} f_{j}\right|^{2}\right)^{1 / 2}\right\|_{L^{p}(w)} \leq C\left\|\left(\sum_{j}\left|f_{j}\right|^{2}\right)^{1 / 2}\right\|_{L^{p}(w)}
$$

holds, then $w^{\theta} \in W_{p}\left(T_{\Omega}\right)$ for $0 \leq \theta<1$. Also, (4) holds if $w \in W_{p}\left(M_{\Omega}\right)$ and $p \leq 2$.

Proof. Decompose $T_{\Omega}$ as before. Then we have the following chain of inequalities:

$$
\begin{aligned}
\left\|\widetilde{T}_{k} f\right\|_{L^{p}(w)} & \leq C\left\|\left(\sum_{j}\left|T_{j} S_{j+k} f\right|^{2}\right)^{1 / 2}\right\|_{L^{p}(w)} \\
& \leq C\left\|\left(\sum_{j}\left|S_{j+k} f\right|^{2}\right)^{1 / 2}\right\|_{L^{p}(w)} \leq C\|f\|_{L^{p}(w)},
\end{aligned}
$$

where the first and third inequalities are deduced from the weighted LittlewoodPaley theory with $w \in A_{p}$ (see $[\mathrm{K}]$ ), and the second inequality is a consequence of $\left|T_{j} f\right| \leq E_{j}(|f|)$ and the hypothesis (4). Using Stein and Weiss' interpolation theorem with change of measure (see [BL, p. 115]) we interpolate with (3) and summing in $k$ we get the first part of the lemma. 
If $w \in W_{p}\left(M_{\Omega}\right)$, then the inequality

$$
\left\|\left(\sum_{j}\left|M_{\Omega} f_{j}\right|^{p}\right)^{1 / p}\right\|_{L^{p}(w)} \leq C\left\|\left(\sum_{j}\left|f_{j}\right|^{p}\right)^{1 / p}\right\|_{L^{p}(w)}
$$

is trivial. Moreover, since $M_{\Omega}$ is a positive operator,

$$
\sup _{j}\left|M_{\Omega} f_{j}(x)\right| \leq M_{\Omega}\left(\sup _{j}\left|f_{j}\right|\right)(x)
$$

pointwise and therefore also in the $L^{p}(w)$-norm. If $p<2$, one can interpolate (see [GR, p. 481] for the vector-valued interpolation) to get (4) with $M_{\Omega}$ instead of $E_{j}$ which is enough.

Lemma 2. If $w \in W_{p^{\prime}}\left(T_{\Omega}\right)$, then $w^{1-p} \in W_{p}\left(T_{\Omega}\right)$.

The lemma is easily proved by duality since $T_{\Omega}$ is (essentially) selfadjoint.

Given $\Omega$, we define $\Omega_{0}(u)=|\Omega(u)|-\|\Omega\|_{1} /\left|S^{n-1}\right|$. Then $\Omega_{0}$ is in the same $L^{q}$ class as $\Omega$ and has mean value zero. The pointwise inequality

$$
E_{j}|f|(x) \leq C T_{j}^{0}|f|(x)+C M f(x)
$$

holds, where $T_{j}^{0}$ is defined as $T_{j}$ but with $\Omega_{0}$ instead of $\Omega$. Then

$$
M_{\Omega} f(x) \leq C g_{\Omega_{0}}(|f|)(x)+C M f(x),
$$

which proves the following lemma.

Lemma 3. If $w \in A_{p} \cap W_{p}\left(g_{\Omega_{0}}\right)$, then $w \in W_{p}\left(M_{\Omega}\right)$.

We obtain weighted inequalities for the square function $g_{\Omega}$ by considering the linear operators $T_{\varepsilon, \Omega} f=\sum_{j} \varepsilon_{j} T_{j} f$, where $\varepsilon=\left\{\varepsilon_{j}\right\}$ is a sequence with $\varepsilon_{j}=$ +1 or -1 . If $T_{\varepsilon, \Omega}$ is bounded in $L^{p}(w)$ with a constant independent of $\varepsilon$, then the usual argument with Rademacher functions [S] gives the boundedness of $g_{\Omega}$ in $L^{p}(w)$. In practice, $T_{\varepsilon, \Omega}$ is decomposed as $T_{\Omega}$ and (3) still holds.

Lemma 4. Let $q<2$ and $p>2$. If $M_{\Omega^{2-q}}$ is bounded in $L^{(p / 2)^{\prime}}\left(w^{1 /(1-p / 2)}\right)$, then the vector-valued inequality (4) in Lemma 1 holds for $p$.

Proof. Applying the Cauchy-Schwarz inequality we get

$$
\left|E_{j} f_{j}(x)\right|^{2} \leq C 2^{-j n} \int_{2^{j}<|x-y| \leq 2^{j+1}}\left|\Omega\left((x-y)^{\prime}\right)\right|^{2-q}\left|f_{j}(y)\right|^{2} d y,
$$

and, if $p>2$, there exists $u$ with unit norm in $L^{(p / 2)^{\prime}}(w)$ such that

$$
\left\|\left(\sum_{j}\left|E_{j} f_{j}\right|^{2}\right)^{1 / 2}\right\|_{L^{p}(w)}^{2}=\int \sum_{j}\left|E_{j} f_{j}(x)\right|^{2} u(x) w(x) d x .
$$


Majorizing $\left|E_{j} f_{j}(x)\right|^{2}$ by the above bound and integrating first in $x$ we have

$$
\begin{aligned}
& \left\|\left(\sum_{j}\left|E_{j} f_{j}\right|^{2}\right)^{1 / 2}\right\|_{L^{p}(w)}^{2} \leq C \int \sum_{j}\left|f_{j}(y)\right|^{2} M_{\Omega^{2-q}}(u w)(y) d y \\
& \quad \leq C\left\|\left(\sum_{j}\left|f_{j}\right|^{2}\right)^{1 / 2}\right\|_{L^{p}(w)}^{2}\left(\int\left|M_{\Omega^{2-q}}(u w)\right|^{(p / 2)^{\prime}} w^{1 /(1-p / 2)}\right)^{1 /(p / 2)^{\prime}},
\end{aligned}
$$

and it suffices to apply the hypothesis of the lemma to see that the last term is bounded.

\section{WeightS IN MUCKeNHOUPT CLASSES}

Theorem 5. (i) $A_{p / q^{\prime}} \subset W_{p}\left(M_{q}\right) \cap W_{p}\left(T_{q}\right)$ if $q^{\prime} \leq p<\infty$;

(ii) $w^{1-p} \in W_{p}\left(M_{q}\right) \cap W_{p}\left(T_{q}\right)$ if $w \in A_{p^{\prime} / q^{\prime}}$ and $1<p \leq q$.

Proof. From (2) and the weighted inequalities for the Hardy-Littlewood maximal function one gets $A_{p / q^{\prime}} \subset W_{p}\left(M_{q}\right)$ if $q^{\prime}<p<\infty$. Since $M_{\Omega}$ is bounded in $L^{p}(w) \forall w \in A_{1}$ for $p>q^{\prime}$, and in $L^{p}$ for $p<q^{\prime}$, interpolation with change of measure (see [BL, p. 119]) gives $A_{1} \subset W_{q^{\prime}}\left(M_{q}\right)$. Here and in the sequel we use the basic fact that

$$
w \in A_{p} \Rightarrow w^{1+\varepsilon} \in A_{p} \quad \text { for some } \varepsilon>0,
$$

which implies $\left\{w^{\theta} / w \in A_{p}, 0 \leq \theta<1\right\}=A_{p}$.

Let $q \geq 2$. Applying Lemma 1 we deduce $A_{p / q^{\prime}} \subset W_{p}\left(T_{q}\right)$ in $q^{\prime} \leq p \leq 2$ and for $p>2$ we can use the extrapolation theorem of Rubio de Francia [R, Theorem 3, p. 539]. In this case, the part of (ii) corresponding to $W_{p}\left(T_{q}\right)$ follows from (i) and Lemma 2. To get (ii) for $W_{p}\left(M_{q}\right)$ we apply Lemma 3. In fact, the weighted inequalities for $T_{\Omega}$ are also valid for $T_{\varepsilon, \Omega}$, and duality can be used for these operators as in Lemma 2 .

Assume now that $\frac{4}{3} \leq q<2$. Then, if $\Omega \in L^{q}\left(S^{n-1}\right)$, we have $\Omega^{2-q} \in$ $L^{q /(2-q)}\left(S^{n-1}\right)$ and $2<q /(2-q)$ so that we can apply the preceding results to $\Omega^{2-q}$. Due to the fact that $(q /(2-q))^{\prime}=q^{\prime} / 2$, when $p \geq q^{\prime}$ we have $w^{1 /(1-p / 2)} \in W_{(p / 2)^{\prime}}\left(M_{\Omega^{2-q}}\right)$ if $w \in A_{p / q^{\prime}}$, according to (ii). Then Lemma 4 applies together with Lemma 1 and (5) to give $A_{p / q^{\prime}} \subset W_{p}\left(T_{q}\right)$. Reasoning as above, Lemmas 2 and 3 provide (ii).

Next we consider the values of $q$ in the range $\frac{8}{7} \leq q<\frac{4}{3}$ for which $\frac{4}{3} \leq$ $q /(2-q)<2$. The results just proved are then valid for $\Omega^{2-q}$ and the same reasoning works. A bootstrapping argument is then applied to get the theorem for any $q>1$.

We do not know whether the analogue of $(5)$ is true for $W_{p}\left(M_{\Omega}\right)$, i.e.,

$$
w \in W_{p}\left(M_{\Omega}\right) \Rightarrow w^{1+\varepsilon} \in W_{p}\left(M_{\Omega}\right) \text { for some } \varepsilon>0
$$

(analogously for $W_{p}\left(M_{q}\right)$ ). Also a duality result like Lemma 2,

$$
w \in W_{p^{\prime}}\left(M_{q}\right) \Rightarrow w^{1-p} \in W_{p}\left(M_{q}\right),
$$


would have avoided the use of the bootstrapping argument. Both properties together would give $W_{p}\left(T_{q}\right)=W_{p}\left(M_{q}\right)$.

More weights can be obtained by interpolating those in (i) and (ii).

\section{RADIAL WEIGHTS}

Theorem 6. $M_{\Omega}$ and $T_{\Omega}$ are bounded in $L^{p}\left(|x|^{\alpha}\right)$ if

$$
\max \left(-n,-1-(n-1) \frac{p}{q^{\prime}}\right)<\alpha<\min \left(n(p-1), p-1+(n-1) \frac{p}{q^{\prime}}\right) .
$$

Proof. Assume $\alpha \leq 0$. When $p \geq q^{\prime}$, the range $-n<\alpha \leq 0$ is given by Theorem 5 since those weights are in $A_{1}\left(\mathbf{R}^{n}\right)$; but they can also be obtained with the method of rotations. Take then $p<q^{\prime}$. Denoting by $M_{u}$ the HardyLittlewood maximal function in the direction $u \in S^{n-1}$ and applying Hölder's inequality we have

$$
\begin{aligned}
M_{\Omega} f(x) & \leq \int_{S^{n-1}}|\Omega(u)| M_{u} f(x) d \sigma(u) \\
& \leq\|\Omega\|_{L^{q}\left(S^{n-1}\right)}^{1 / p^{\prime}}\left(\int_{S^{n-1}}|\Omega(u)|^{\left(1-q / p^{\prime}\right) p}\left|M_{u} f(x)\right|^{p} d \sigma(u)\right)^{1 / p} .
\end{aligned}
$$

Then

$$
\begin{aligned}
\int_{\mathbf{R}^{n}} \mid & \left.M_{\Omega} f(x)\right|^{p} w(x) d x \\
& \leq C \int_{S^{n-1}}|\Omega(u)|^{\left(1-q / p^{\prime}\right) p} \int_{\mathbf{R}^{n}}\left|M_{u} f(x)\right|^{p} w(x) d x d \sigma(u) \\
& \leq C \int_{S^{n-1}}|\Omega(u)|^{\left(1-q / p^{\prime}\right) p} \int_{\mathbf{R}^{n}}|f(x)|^{p} M_{u} w(x) d x d \sigma(u)
\end{aligned}
$$

(see [GR, p. 150] for the last inequality) so that $w \in W_{p}\left(M_{\Omega}\right)$ whenever

$$
\int_{S^{n-1}}|\Omega(u)|^{\left(1-q / p^{\prime}\right) p} M_{u} w(x) d \sigma(u) \leq C w(x) \quad \text { a.e. }
$$

Applying Hölder's inequality again with exponents $q /\left(1-q / p^{\prime}\right) p=1 /(1-$ $\left.p / q^{\prime}\right)$ and its conjugate $q^{\prime} / p$, it is enough to prove

$$
\left(\int_{S^{n-1}}\left|M_{u} w(x)\right|^{q^{\prime} / p} d \sigma(u)\right)^{p / q^{\prime}} \leq C w(x) \text { a.e. }
$$

Take $w(x)=|x|^{\alpha}$ with $\alpha<0$. Since $w$ is radial, the left-hand side of (6) is also radial and we can assume $x=(|x|, 0, \ldots, 0)$. It is easy to compute $M_{u} w(x)$ because the restriction of $w$ to the line through $x$ with direction $u$ is symmetric and decreasing with respect to the projection of the origin on this line. So, if $\theta$ is the angle of $u$ with the $x_{1}$ axis, we get

$$
\begin{aligned}
M_{u} w(x) & \sim C|x|^{\alpha} \quad \text { when }|\sin \theta| \geq|\cos \theta| \text { or }-1<\alpha \leq 0 \\
& \sim C|x|^{\alpha}|\sin \theta|^{1+\alpha} \quad \text { when }|\sin \theta| \leq|\cos \theta| \text { and } \alpha<-1 ; \\
& \sim C|x|^{\alpha} \log \frac{1}{|\sin \theta|} \quad \text { when }|\sin \theta| \leq|\cos \theta| \text { and } \alpha=-1,
\end{aligned}
$$

and (6) holds whenever

$$
\int_{0}^{\pi / 4}(\sin \theta)^{(1+\alpha) q^{\prime} / p}(\sin \theta)^{n-2} d \theta<+\infty
$$


which is true if $-1-(n-1) p / q^{\prime}<\alpha$, as the hypothesis of the theorem states.

For $T_{\Omega}$, the values $-n<\alpha \leq 0$ for $p \geq q^{\prime}$ are still given by Theorem 5 , and Lemma 1 applies when $1<p \leq \min \left(2, q^{\prime}\right)$. If $2<q^{\prime}$, then one applies interpolation to fill the gap $2<p<q^{\prime}$. The result for $\alpha>0$ is given by duality (Lemma 2). To apply the duality to $M_{\Omega}$ we pass through Lemma 3 as above.

Theorem 6 was proved for $T_{\Omega}$ by Muckenhoupt and Wheeden [MW] in a different way and they also showed that the range is optimal. Their method also works for $M_{\Omega}$. Our approach is based on the method of rotations and shows that any operator given as

$$
\int_{S^{n-1}} \Omega(u) R_{u} f(x) d \sigma(u),
$$

where $R_{u}$ denotes the directional operator defined from some one-dimensional operator $R$ bounded in $L^{p}(v, \mathbf{R})$ for all $v \in A_{1}(\mathbf{R})$, is bounded in $L^{p}(w)$ for all $w$ satisfying (6) with $\left(M_{u} w^{s}\right)^{1 / s}$ (any $s>1$ ) instead of $M_{u} w$. This modification comes from the fact that one has to use the inequality

$$
\int\left|R_{u} f(x)\right|^{p} w(x) d x \leq C \int|f(x)|^{p}\left(M_{u} w^{s}(x)\right)^{1 / s} d x
$$

with $s>1$ (since $\left(M_{u} w^{s}\right)^{1 / s}$ is an $A_{1}$-weight) instead of the one used in the proof of the theorem with $s=1$, which is false in general (for example if $R$ is the Hilbert transform).

Recalling that $|x|^{\alpha} \in A_{p}\left(\mathbf{R}^{n}\right)$ if and only if $-n<\alpha<n(p-1)$, one can verify that there are more power weights in Theorem 6 than in Theorem 5.

Theorem 7. Let $w(x)=v_{1}(|x|) v_{2}^{1-p}(|x|)$, where either $v_{i} \in A_{1}\left(\mathbf{R}^{+}\right)$and is decreasing or $v_{i}^{2} \in A_{1}\left(\mathbf{R}^{+}\right), i=1,2$. Then $M_{\Omega}$ and $T_{\Omega}$ are bounded in $L^{p}(w)$. This result is valid for $M_{\Omega}$ when $\Omega \in L^{1}\left(S^{n-1}\right)$, and also for $T_{\Omega}$ if moreover $\Omega$ is odd.

Proof. For a radial function $v$ such that $v(x)=v_{0}(|x|)$ it is shown in [CHS] that

$$
M_{u} v(x) \leq C\left(M v_{0}^{2+\delta}(|x|)\right)^{1 /(2+\delta)} \quad \forall \delta>0
$$

and, if $v$ is decreasing,

$$
M_{u} v(x) \leq C M v_{0}(|x|),
$$

where $M$ is the Hardy-Littlewood maximal function on $\mathbf{R}^{+}$. With the assumptions of the theorem, $v_{i}(|x|), i=1,2$, satisfies the $A_{1}$ condition for $M_{u}$ uniformly in $u$ so that $w(x)=v_{1}(|x|) v_{2}^{1-p}(|x|)$ satisfies the $A_{p}$ condition uniformly. Then

$$
\int\left|M_{u} f(x)\right|^{p} w(x) d x \leq C \int|f(x)|^{p} w(x) d x
$$

with $C$ independent of $u$.

The theorem is now immediate for $M_{\Omega}$ since

$$
\left\|M_{\Omega} f\right\|_{L^{p}(w)} \leq \int_{S^{n-1}}|\Omega(u)|\left\|M_{u} f\right\|_{L^{p}(w)} d \sigma(u) .
$$


Applying (8) with the Hilbert transform instead of $M$ we get the theorem for $T_{\Omega}$ when $\Omega$ is odd. When $\Omega$ is even, the usual modification of the method of rotations (see [CZ]) does the job.

According to P. Jones' factorization theorem [GR, p.436] the weights $w(x)=$ $w_{0}(|x|)$ such that $w_{0}^{2} \in A_{p}\left(\mathbf{R}^{+}\right)$are included in the above statement. For power weights $|x|^{\alpha}$ this theorem only gives $-1<\alpha<p-1$ which is the best possible result for $\Omega \in L^{1}\left(S^{n-1}\right)$ (not included in Theorem 6).

Again the result can be generalized to all operators represented as in (7) provided $R$ is bounded in $L^{p}(v, \mathbf{R})$ for all $v \in A_{p}(\mathbf{R})$.

Proceeding as in Theorem 7 one can generalize the results of [DR2]:

Theorem 8. Let $\left\{\boldsymbol{R}_{j}\right\}_{j=1}^{n}$ be the $n$ Riesz transforms in $\mathbf{R}^{n}$, and $w$ a radial weight of those appearing in Theorem 7. Then, for $1<p<\infty$, there exists a constant $C$ depending only on $w_{0}$ and $p$ and not on $n$ such that

$$
\left\|\left(\sum_{j=1}^{n}\left|R_{j} f\right|^{2}\right)^{1 / 2}\right\|_{L^{p}(w)} \leq C\|f\|_{L^{p}(w)} .
$$

An analogous theorem exists for higher order Riesz transforms. All one needs is to implement into the proofs of [DR2] the weighted inequality (8) (with the Hilbert transform) and realize that the constant in it is independent of $n$.

Let us finally indicate that some radial weights are also obtained for singular integrals with variable kernel:

$$
T f(x)=\text { p.v. } \int_{\mathbf{R}^{n}} \frac{\Omega\left(x, y^{\prime}\right)}{|y|^{n}} f(x-y) d y,
$$

where $\sup _{x}\|\Omega(x, \cdot)\|_{L^{q}\left(S^{n-1}\right)}<+\infty$. Theorem 7 holds with $p \geq q^{\prime}$ and Theorem 6 holds with $-n<\alpha \leq 0$ and $p \geq q^{\prime}$ (a better range appears in [MW]). The details are left to the reader.

\section{WEIGHTS DEPENDING ON $\Omega$}

The spirit of the extrapolation theorem is that the weighted $L^{2}$-inequalities, for example, contain all the information about the weighted $L^{p}$-inequalities. A weaker (and much easier) statement would be that the weighted $L^{2}$-inequalities contain the unweighted $L^{p}$-inequalities. Assuming $p>2$, for instance, for some $u \in L^{(p / 2)^{\prime}}\left(\mathbf{R}^{n}\right)$ of unit norm we have

$$
\left\|T_{\Omega} f\right\|_{p}^{2}=\int\left|T_{\Omega} f\right|^{2} u
$$

and one only needs $w \in W_{2}\left(T_{\Omega}\right)$ such that $u \leq w$ a.e. and $\|w\|_{(p / 2)^{\prime}} \leq$ $C\|u\|_{(p / 2)^{\prime}}$ in order to deduce the $L^{p}$ boundedness of $T_{\Omega}$ in a standard way. When $\Omega \in L^{q}\left(S^{n-1}\right)$ and $q \geq 2$, Theorem 5 assures that $A_{1} \subset W_{2}\left(T_{\Omega}\right)$ and $w=\left(M u^{s}\right)^{1 / s}$ for any $s$ between 1 and $(p / 2)^{\prime}$ does the job. But if $q<2$, none of the above theorems provides enough weights to deduce the $L^{p}$-inequalities. However, we know that $T_{\Omega}$ is bounded in $L^{p}, 1<p<\infty$. The restriction on the amount of weights comes from the fact that we have been producing weights valid for all $\Omega \in L^{q}$, rather than for a particular one, and the purpose of the following theorem is to show how to construct weights in $W_{2}\left(T_{\Omega}\right)$ such that the $L^{p}$-inequalities follow. 
Theorem 9. Let $\Omega \in L^{q}\left(S^{n-1}\right), q<2,1<s<r<\infty, u \in L^{r}\left(\mathbf{R}^{n}\right)$, and $A$ be the norm of the operator $M_{\Omega^{2-q}} M$ in $L^{r / s}\left(\mathbf{R}^{n}\right)$. Then

$$
w=\left(\sum_{n=0}^{\infty} \frac{1}{(2 A)^{n}}\left(M_{\Omega^{2-q}} M\right)^{n} u^{s}\right)^{1 / s} \in W_{2}\left(T_{\Omega}\right),
$$

where $\left(M_{\Omega^{2-q}} M\right)^{n}$ stands for the composition $n$ times of $M_{\Omega^{2-q}} M$.

Proof. We claim that inequality (4) in Lemma 1 holds when $p=2$ for any function $w$ satisfying $M_{\Omega^{2-q}} w \leq C w$ a.e. In fact, proceeding as in Lemma 4 (with $p=2$, so that $u \equiv 1$ ),

$$
\int \sum_{j}\left|E_{j} f_{j}\right|^{2} w \leq C \int \sum_{j}\left|f_{j}\right|^{2} M_{\Omega^{2-q}} w .
$$

The series in the statement of the theorem converges in the norm of $L^{r / s}\left(\mathbf{R}^{n}\right)$ and since $M$ and $M_{\Omega^{2-q}}$ are positive operators with $v \leq \min \left(M v, M_{\Omega^{2-q}} v\right)$ we have

$$
\max \left(M w^{s}, M_{\Omega^{2-q}} w^{s}\right) \leq M_{\Omega^{2-q}} M w^{s} \leq \sum_{n=0}^{\infty} \frac{1}{(2 A)^{n}}\left(M_{\Omega^{2-q}} M\right)^{n+1} u^{s} \leq 2 A w^{s}
$$

so that $w^{s}$ is an $A_{1}$-weight (hence $A_{2}$ ), which satisfies $M_{\Omega^{2-q}} w^{s} \leq 2 A w^{s}$. By applying Lemma 1 with $w^{s}$ in the inequality (4) we deduce that $w \in$ $W_{2}\left(T_{\Omega}\right)$.

The construction of $w$ in the theorem follows from the algorithm of Rubio de Francia in [R]. It is clear that it satisfies $u \leq w$ a.e. (just taking $n=0$ in the series) and $\|w\|_{r} \leq C\|u\|_{r}$, the conditions required above to deduce the $L^{p}$-inequalities with $p>2$.

\section{THE MAXIMAL SINGULAR INTEGRAL}

We define the following maximal operator associated to the singular integral $T_{\Omega}$ :

$$
T_{\Omega}^{*} f(x)=\sup _{\varepsilon \rightarrow 0}\left|T^{\varepsilon} f(x)\right|=\sup _{\varepsilon \rightarrow 0}\left|\int_{|y|>\varepsilon} \frac{\Omega\left(y^{\prime}\right)}{|y|^{n}} f(x-y) d y\right| .
$$

It is well known that the boundedness of $T_{\Omega}^{*}$ in $L^{p}(w)$ implies the almost everywhere existence of $\lim _{\varepsilon \rightarrow 0} T^{\varepsilon} f(x)$, the principal value integral defining $T_{\Omega}$ for $f \in L^{p}(w)$. We apply again the method of [DR1] or the method of rotations in order to get weighted norm inequalities for $T_{\Omega}^{*}$.

Theorem 10. (i) Let $\Omega \in L^{q}\left(S^{n-1}\right), q>1$, let $1<p<\infty$, and let $w$ be as in Theorem 5, 6, or 7. Then $T_{\Omega}^{*}$ is bounded in $L^{p}(w)$.

(ii) Let $\Omega \in L^{1}\left(S^{n-1}\right)$ be odd. Then $T_{\Omega}^{*}$ is bounded in $L^{p}(w)$ for $w$ as in Theorem 7.

Proof. (i) Assume $2^{k} \leq \varepsilon<2^{k+1}$. Then

$$
\left|T^{\varepsilon} f(x)\right| \leq C M_{\Omega} f(x)+\left|T^{2^{k}} f(x)\right|,
$$

so that it is enough to take dyadic values of $\varepsilon$. On the other hand,

$$
\left|T^{2^{k}} f(x)\right|=\left|\sum_{j=k}^{\infty} T_{j} f\right| \leq C M\left(T_{\Omega} f\right)+C M f+\left|\left(\delta-\Phi_{k}\right) * \sum_{j=k}^{\infty} T_{j} f\right|
$$


is the inequality proved in [DR 1$]$ with $\delta$ the Dirac delta and $\Phi_{k}=2^{-k n} \Phi\left(2^{-k} \cdot\right)$, where $\Phi$ is in the Schwartz class $\mathscr{S}\left(\mathbf{R}^{n}\right), \widehat{\Phi}(0)=1$, and $\operatorname{supp} \Phi \subset B(0,1)$. Therefore, it is enough to consider the last term, which in turn is bounded by

$$
\sup _{k}\left|\left(\delta-\Phi_{k}\right) * \sum_{j=k}^{\infty} T_{j} f\right| \leq \sum_{j=0}^{\infty} \sup _{k}\left|\left(\delta-\Phi_{k}\right) * T_{j+k} f\right| .
$$

Each term in the sum is bounded by $\sup _{k}\left|\left(\delta+|\Phi|_{k}\right) * M_{\Omega} f\right|$ so that all the weights obtained for $M_{\Omega}$ are valid for it. Moreover, in any unweighted $L^{p_{0}}$ norm it has an exponential decay (use the Fourier transform in $p_{0}=2$ and interpolate for other values).

(ii) When $\Omega$ is odd we have

$$
T^{\varepsilon} f(x) \leq \pi \int_{S^{n-1}}|\Omega(u)|\left|H_{u}^{*} f(x)\right| d \sigma(u),
$$

where $H^{*}$ is the maximal Hilbert transform. The right-hand side of the inequality is an operator of the form (7) so that the proof of Theorem 7 applies to it.

When $\Omega$ is even and belongs to $L^{q}\left(S^{n-1}\right), q>1$, the method of rotations can be applied by using several operators of the type (7) because $T_{\Omega}$ has to be written as a combination of Riesz transforms applied to operators with odd kernels (see [CZ] for the details). The method used in (ii) is then applicable to these operators and we get the weights in Theorems 6 and 7.

\section{SOME EXTENSIONS AND REMARKS}

(a) Theorems 5 and 9 can be extended to the case where the kernel of the singular integral contains a radial bounded function $h$, i.e.,

$$
T f(x)=\lim _{\varepsilon \rightarrow 0} \int_{|y|>\varepsilon} \frac{h(|y|) \Omega\left(y^{\prime}\right)}{|y|^{n}} f(x-y) d y .
$$

These operators were studied by Fefferman in $[\mathrm{F}]$ by using interpolation of an analytic family of operators, but it was shown in [DR1] that the method sketched in $\S 2$ of this paper also works. Again this method gives Theorems 5 and 9 and the corresponding part of Theorem 10. However, since the method of rotations is not applicable here, we do not have the analogues of Theorems 6 and 7.

(b) Another modification concerns the use of a pseudonorm associated to a nonisotropic group of dilations in $\mathbf{R}^{n}$ instead of the euclidean norm, i.e.,

$$
T f(x)=\lim _{\varepsilon \rightarrow 0} \int_{\|y\|>\varepsilon} \frac{h(\|y\|) \Omega\left(y^{\prime}\right)}{\|y\|^{a}} f(x-y) d y,
$$

where $\|\cdot\|$ denotes the pseudonorm, $a$ is the homogeneous dimension of $\mathbf{R}^{n}$ with respect to this group, $h \in L^{\infty}(0, \infty)$, and $\Omega \in L^{q}\left(S^{n-1}\right), q>1$. The definition of the $A_{p}$-class of weights must be modified according to the present structure of $\mathbf{R}^{n}$ in the standard way. Here, even if $h \equiv 1$, the method of rotations cannot be applied because the directional operators would correspond to maximal functions and Hilbert transforms along homogeneous curves, for 
which the appropriate weighted inequalities are still unknown. So, we again get Theorems 5 and 9 and part of Theorem 10, and miss Theorems 6 and 7.

(c) Multiple singular integrals in product spaces and strong maximal operators can also be treated by the methods in this paper. By using products of two spaces, $\mathbf{R}^{n} \times \mathbf{R}^{m}$, in order to simplify notations, the operators are

$$
T_{\Omega} f\left(x_{1}, x_{2}\right)=\lim _{\varepsilon_{1}, \varepsilon_{2} \rightarrow 0} \int_{\substack{\left|y_{1}\right|>\varepsilon_{1} \\\left|y_{2}\right|>\varepsilon_{2}}} \frac{\Omega\left(y_{1}^{\prime}, y_{2}^{\prime}\right)}{\left|y_{1}\right|^{n}\left|y_{2}\right|^{m}} f\left(x_{1}-y_{1}, x_{2}-y_{2}\right) d y_{1} d y_{2}
$$

and

$$
M_{\Omega} f\left(x_{1}, x_{2}\right)=\sup _{r_{1}, r_{2}>0} \frac{1}{r_{1}^{n} r_{2}^{m}} \int_{\substack{\left|y_{1}\right|<r_{1} \\\left|y_{2}\right|<r_{2}}}\left|\Omega\left(y_{1}^{\prime}, y_{2}^{\prime}\right)\right|\left|f\left(x_{1}-y_{1}, x_{2}-y_{2}\right)\right| d y_{1} d y_{2} .
$$

Muckenhoupt's definition of $A_{p}$ in $\S 1$ is now modified by taking averages over products of cubes (one gets the weights for the strong maximal function, see [GR]). Looking at [D], where the unweighted inequalities were obtained, the reader will easily find the way to modify Lemmas 1 and 4 and Theorems 5 and 9. As in the unweighted case we are not able to handle the maximal singular integral.

Theorems 6 and 7 are extended to $M_{\Omega}$ for any intregrable $\Omega$ and to $T_{\Omega}$ for $\Omega$ odd in both variables by using the method of rotations and biradial weights, i.e., $w(x)=\left|x_{1}\right|^{\alpha_{1}}\left|x_{2}\right|^{\alpha_{2}}$ with $\alpha_{1}$ and $\alpha_{2}$ in the appropriate range in Theorem 6 and $w(x)=w_{1}\left(\left|x_{1}\right|\right) w_{2}\left(\left|x_{2}\right|\right)$ with $w_{1}, w_{2}$ as in Theorem 7. For these weights $T_{\Omega}^{*}$ is also bounded. When $\Omega$ is not odd in both variables, the method of rotations seems difficult to apply even in the unweighted case.

(d) If we define $W_{1}\left(M_{\Omega}\right)$ as

$$
W_{1}\left(M_{\Omega}\right)=\left\{w / M_{\Omega} w \leq C w \text { a.e. }\right\}
$$

then any weight $w \in W_{p}\left(M_{\Omega}\right)$ such that $w^{1-p^{\prime}} \in W_{p^{\prime}}\left(M_{\Omega}\right)$ (in particular, all those appearing in our theorems) can be factorized as $w=w_{0} w_{1}^{1-p}, w_{0}, w_{1} \in$ $W_{1}\left(M_{\Omega}\right)$ (see $\left.[\mathrm{R}]\right)$. If we were able to prove the duality property mentioned in $\S 3$, namely

$$
w \in W_{p}\left(M_{\Omega}\right) \Leftrightarrow w^{1-p^{\prime}} \in W_{p^{\prime}}\left(M_{\Omega}\right),
$$

then any weight in $W_{p}\left(M_{\Omega}\right)$ could be factorized as before.

(e) Weak-type $(1,1)$ weighted inequalities have been studied for $T_{\Omega}$ in [KW1, KW2] and for $T_{\Omega}^{*}$ in [CS], in both cases by assuming a Dini-type condition on $\Omega$. The situation is much more complicated when we merely assume a size condition on $\Omega$, as is shown by the fact that even the unweighted weaktype estimate was obtained only thirty years after the $L^{p}$ one was proved (see $[\mathrm{C}, \mathrm{CR}, \mathrm{H} 1])$. One could conjecture that the inequality

$$
w\left(\left\{x:\left|T_{\Omega} f(x)\right|>\lambda\right\}\right) \leq \frac{C}{\lambda} \int|f| w
$$

is satisfied when either $w(x)=|x|^{\alpha}$ with $-1-(n-1) / q^{\prime}<\alpha \leq 0$ (from Theorem 6), or when $w(x)=w_{0}(|x|)$ and either $w_{0} \in A_{1}\left(\mathbf{R}^{+}\right)$and is decreasing or $w_{0}^{2} \in A_{1}\left(\mathbf{R}^{+}\right)$(from Theorem 7 ). Also, a similar conjecture could be made for $M_{\Omega}$. In the case of power weights and $n=2$, this result was proved by Hofmann [H2]. The conjecture from Theorem 5 is that (9) holds when $w^{q^{\prime}} \in A_{1}\left(\mathbf{R}^{n}\right)$. 
(f) When $\Omega \in L \log ^{+} L\left(S^{n-1}\right), T_{\Omega}$ is bounded in $L^{p}(w)$ for $w(x)=|x|^{\alpha}$, $-1<\alpha<p-1$, and also for $w$ as in Theorem 7 by using the method of rotations. Also $T_{\Omega}$ is bounded in $L^{2}(w)$ for $w$ as in Theorem 9 with $q=1$. In this case, one can extrapolate from the result for $q>1$ since the constant has a size of the order $\|\Omega\|_{q}(q-1)^{-1}$.

\section{REFERENCES}

[BL] J. Bergh and J. Löfström, Interpolation spaces. An introduction, Springer-Verlag, Berlin, 1976.

[CZ] A. P. Calderón and A. Zygmund, On singular integrals, Amer. J. Math. 78 (1956), 289-309.

[CS] O. N. Capri and C. Segovia, Behaviour of $L^{r}$-Dini singular integrals in weighted $L^{1}$-spaces, Studia Math. 92 (1989), 21-36.

[CHS] A. Carbery, E. Hernández, and F. Soria, Estimates for the Kakeya maximal operator on radial functions in $\mathbb{R}^{n}$, Harmonic Analysis, ICM-90 Satellite Conference Proceedings, S. Igari (ed.), Springer-Verlag, Tokyo, 1991, pp. 41-50.

[C] M. Christ, Weak type $(1,1)$ bounds for rough operators, Ann. of Math. (2) 128 (1988), 19-42.

[CR] M. Christ and J. L. Rubio de Francia, Weak type $(1,1)$ bounds for rough operators. II, Invent. Math. 93 (1988), 225-237.

[D] J. Duoandikoetxea, Multiple singular integrals and maximal functions along hypersurfaces, Ann. Inst. Fourier (Grenoble) 36 (1986), 185-206.

[DR1] J. Duoandikoetxea and J. L. Rubio de Francia, Maximal and singular integral operators via Fourier transform estimates, Invent. Math. 84 (1986), 541-561.

[DR2] __ Estimations indépendantes de la dimension pour les transformées de Riesz, C. R. Acad. Sci. Paris 300 (1985), 193-196.

[F] R. Fefferman, A note on singular integrals, Proc. Amer. Math. Soc. 74 (1979), 266-270.

[GR] J. Garcia-Cuerva and J. L. Rubio de Francia, Weighted norm inequalities and related topics, North-Holland, Amsterdam, 1985.

[H1] S. Hofmann, Weak $(1,1)$ boundedness of singular integrals with nonsmooth kernel, Proc. Amer. Math. Soc. 103 (1988), 260-264.

[H2] Weighted weak-type $(1,1)$ inequalities for rough operators, Proc. Amer. Math. Soc. 107 (1989), 423-435.

[K] D. S. Kurtz, Littlewood-Paley and multiplier theorems on weighted $L^{p}$ spaces, Trans. Amer. Math. Soc. 259 (1980), 235-254.

[KW1] D. S. Kurtz and R. L. Wheeden, Results on weighted norm inequalities for multipliers, Trans. Amer. Math. Soc. 255 (1979), 343-362.

[KW2] _ A note on singular integrals with weights, Proc. Amer. Math. Soc. 81 (1981), 391-397.

[MW] B. Muckenhoupt and R. L. Wheeden, Weighted norm inequalities for singular and fractional integrals, Trans. Amer. Math. Soc. 161 (1971), 249-258.

[R] J. L. Rubio de Francia, Factorization theory and $A_{p}$ weights, Amer. J. Math. 106 (1984), 533-547.

[S] E. M. Stein, Singular integrals and differentiability properties of functions, Princeton Univ. Press, Princeton, N. J., 1970.

[W] D. Watson, Weighted estimates for singular integrals via Fourier transform estimates, Duke Math. J. 60 (1990), 389-399.

Departamento de Matemáticas, Universidad Autónoma, 28049 Madrid, Spain

Current address: Departamento de Matemáticas, Universidad del Pais Vasco, Apartado 644, 48080 Bilbao, Spain

E-mail address: mtpduzuj@lg.ehu.es 